\title{
Applying Workflow to Experiment Control in Virtual Laboratory
}

\author{
Łukasz Czekierda and Krzysztof Zieliński \\ Distributed Systems Research Group \\ Department of Computer Science \\ AGH - University of Science and Technology \\ al. Mickiewicza 30, 30-059 Kraków, Poland \\ \{luke, kz\} aics.agh.edu.pl
}

\begin{abstract}
Virtual Laboratory (VLab) has been developed as a distributed component system supporting a remote access to physical devices via the Internet. Unlike in typical VLabs, much attention has been paid to designing a state-ofthe-art architecture which facilitates among others exposing the functionality of the devices, composing them into complex experiment stands as well as supervising in an interactive or automated way. The paper describes an application of workflow to experiment control in the VLab. Nevertheless, the presented concept consisting in wrapping a workflow engine by a component of a selected platform component has much more general nature.
\end{abstract}

\section{Motivation of the Work}

Virtual laboratories become popular nowadays. WWW resources direct us usually to simple simulation programs from various disciplines implemented using Java applets, ActiveX controls, etc. There are also environments which offer users an access to real devices; the paper concentrates on VLabs understood according to such a meaning.

Virtualization technique makes it possible to see the equipment of the laboratory not as a collection of heterogeneous physical devices but as software entities that wrap their functionality and provide with a well-defined interface for remote management and configuration. This technique is widely accepted and does not pose a considerable problem (using such technologies as Web Services), thus access via Internet to devices such as radio-telescopes is currently almost an everyday practice.

Current virtual laboratories are to a great degree static. Devices are often bound with their graphical user interface and sometimes with each other. Users are not able to change the administratively pre-set order of actions and customize the equipment to their needs. Nevertheless, there are a few exceptions to this, e.g. [2, 3].

A next generation VLab should offer much more features. The most crucial requirement is a clear separation of representation of the device functionality (its instrumentation) from usage scenarios. Thanks to the separation, both a single device or set of them consistently interconnected can be controlled in many various ways depending on goals of performed activities, user's permissions etc.

Interface exposed by the instrumented devices is usually tied to GUI used to influence the experiment course. In some cases the same activities could be performed by an 
agent - the user representative. Some experiments can be realized in a mixed mode the routine parts are performed automatically and the user takes a decision in crucial moments. Experiment logic may be programmed by the VLab administrator. However, in most cases it is better to allow users to do it by themselves. Performing the task must be feasible even for people with no much programming skills - thus they must be provided with a proper tool - user-friendly yet powerful. Amongst many techniques available, using workflow seems to be the best solution.

\section{Component-Based Architecture of Virtual Laboratory}

VLab as most of the contemporary distributed systems has a layered architecture.

The lowest layer of the VLab - physical devices layer - is composed of a collection of heterogeneous physical devices. The second layer - instrumentation layer provides devices' representatives exposing a generic management and configuration interface. For representation of virtualized devices CORBA Component Model (CCM) [1] components have been chosen, their interface is expressed using Interface Definition Language (IDL). CCM object definition clearly states which channels (interfaces) the object accepts, which interfaces it uses and which events it emits and consumes, what definitely much better characterizes the object interface than the traditional object-oriented languages.

Thanks to the well-known CCM channel in-

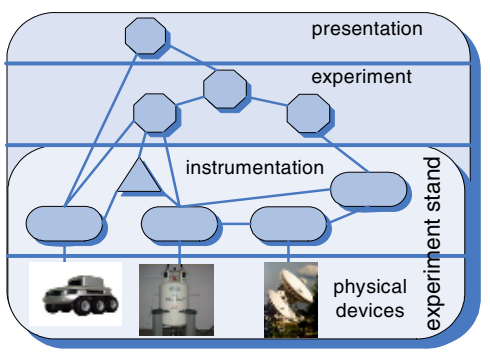

Fig. 1. Multilayer architecture of Virtual Laboratory terface it can be automatically determined whether particular devices can be linked via given channels or not. When the interfaces are not fully compatible, an adapter can be used. This conglomerate can be called the experiment stand and is presented in Fig. 1.

Some channels are devoted to external control - they can be attached to the user elements or to elements playing the role of the user's agents. The user should be allowed to supervise the operation of the agent. Agents and user application constitute two upper layers of the architecture of the Virtual Laboratory - experiment and presentation, respectively. They have been also implemented as CCM components.

\section{Embedding Workflow Engine Inside a Component}

The idea implemented in the VLab has a much more general nature consisting in encapsulating a workflow engine by a component of a given component platform. This concept is illustrated in Fig. 2. The benefits of such approach are following:

- transparency and uniform representation - the object can be seen and used as any other component (including assembling, configuration and deployment). 
- precisely defined interface - workflow environments are able to run any process instance, so the workflow engine lacks a well-defined interface with the external world. Embedding it in the component forces to specify the exact interface.

Achieving the goal is in general not a straightforward undertaking. To the most important issues which need to be considered belong:

- transforming the interface and communication - the concept assumes linking two distinct worlds - external one with RPC-based communication and internal one with other or not specified mechanism. Usually, providing an intermediate layer bridging the communication will be necessary.

- providing the management interface - besides the interface used for communication with external partners, the component ruled by workflow needs an additional channel - for introducing process definition, starting, stopping or repeating it, etc.

- monitoring the workflow - workflow engines can be usually monitored what allows users to e.g. check the progress of the workflow execution.

- evaluating portability and efficiency - workflow engines may need many special libraries and artifacts to run. Moreover, running them with only business process instance requires loading all of the anyway. Thus, lightweight engines are desired.

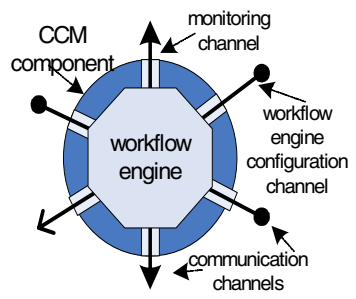

Fig. 2. Placement of workflow engine inside CCM component

In the context of the VLab, the concept has many advantages. All components used in the experiment are uniformly represented and can be assembled together as well as easily scheduled and deployed. With the ability not only to connect required elements but also to define their behavior, users can construct complete experiments.

\section{Implementing the Concept in CCM Component Platform}

From among a few workflow platforms investigated (BPEL, YAWL), Java Business Process Management (jBPM) [5] has been chosen. It easily integrates with chosen CCM platform implementation - java-based openCCM from ObjectWeb.

$\mathrm{jBPM}$ is implemented according to UML activity diagram and is declared to support most of workflow patterns specified by van der Aalst [4]. jBPM combines declarative and procedural approach of process behavior definition - its core is expressed in XML-like jPDL language and actions (handlers) assigned with some events can be written in java. This increases expressiveness of the workflow.

Process definition in jBPM consists of many nodes connected via transitions. Nodes are visited by a token passing from a start to an end node. There are several types of nodes; from the point of view of this discussion two are the most important:

- state nodes - store the token (pointing an active node) until an external event moves it to a next node. State node is then a stable state.

- ordinary nodes - there can be assigned a piece of work to be done (action) to them. The token leaves the node immediately after executing the action. 
jBPM does not directly support remote communication (especially CORBA). In case of outgoing invocations (to external CORBA objects) it is possible to implement handlers assigned with the node which are able to perform any action - in particular do remote CORBA invocations. Incoming communication is much more difficult. In authors' implementation incoming operation invocations are parsed, converted by a special intermediate layer to so called signals and assigned with a proper token. As a result of receiving the signal, the token leaves the current state node, passes through several nodes executing defined actions and stops in the next state node.

It is worth to emphasize roles of the VLab administrator and users in this context. Administrator specifies the component's interface and implements the handlers. The single handler should encapsulate a simple functionality so as to be able to build the behavior of the component in a fine-grained way. Constructing the workflow using existing elements can be done by the users provided with a proper (graphical) tool.

\section{Applying the Concept in the Virtual Laboratory - Case Study}

The presented architecture has been implemented. To the collection of virtualized devices belong mobile robots, a localization system, a video streaming server, and a pan-tilt camera. Several experiments have been designed and implemented using these devices, most of them give full control over the experiment course to the users.

The benefits of using workflow in the VLab are evidently visible especially in case of experiments supervising a technological or computational process where finishing task. Nevertheless, even using the mentioned virtualized devices it is possible to successfully apply workflow to experiment control.

The experiment stand contains the mobile robot and the localization system which observes it. The robot is expected to come from the starting to the end point via several destinations on the experiment area with some obstacles placed. The role of workflow is to lead it so as to avoid obstacles. Nevertheless, hitting one of them triggers notification received by the workflow and forces to return to the previous destination successfully reached. The experiment layer CCM component is connected to: (1) several input channels notifying about reaching a destination, (2) input channel notifying about hitting an obstacle or entering a forbidden area, (3) output management channel of the robot driving its wheels, (4) input management channel coming from the user supervising the experiment and able to start, pause and finish it.

\section{References}

1. Object Management Group. CORBA Components, June 2002, formal/02-06-65.

2. Fjeldly T A. Shur M. S., Lab on the Web, Running Real Electronics Experiments via the Internet, Wiley\&Sons, IEEE Press, 2003.

3. Lawenda M., Meyer N., Workflow with Dynamic Management Scenarios in the Virtual Laboratory, $6^{\text {th }}$ CARNet Users Conference, ISBN 953-6802-04-X, September 27-29, 2004.

4. van der Aalst W.M.P., ter Hofstede A.H., Kiepuszewski B., Barros A., Workflow Patterns.

5. Koenig J. JBoss jBPM, White Paper, November 2004. 